M. Kishi

Nagoya Math. J.

Vol. 48 (1972), 189-196

\title{
AN EXAMPLE OF A POSITIVE NON-SYMMETRIC KERNEL SATISFYING THE COMPLETE MAXIMUM PRINCIPLE
}

\section{Dedicated to Professor Yukinari TÔKI on his sixtieth birthday}

\author{
MASANORI KISHI
}

\section{§1. Introduction and preliminaries.}

One of the main problems in potential theory is to determine the class of kernels satisfying the domination principle or the complete maximum principle. As to positive symmetric kernels this is settled to a certain extent, but as to non-symmetric kernels we have not yet obtain satisfactorily large explicit classes. In this note we shall give a class of positive non-symmetric convolution kernels on the real line satisfying the complete maximum principle.

Let $K$ be a positive Radon measure on the real line $\boldsymbol{R}$. Given an essentially bounded Lebesgue measurable function $f$ vanishing outside a compact set, the convolution $K * f$ is called a potential relative to a positive kernel $K$. This is a Lebesgue measurable function on $\boldsymbol{R}$. Throughout this note we shall identify two functions which differ on a set of Lebesgue measure zero.

We shall say that the kernel $K$ satisfies the complete maximum principle, when the following statement is valid: if $f$ and $g$ are essentially bounded Lebesgue measurable functions vanishing outside a compact set and $K * f \leqq K * g+1$ on the set $\{x \in R ; f(x)>0\}$, then the same inequality holds almost everywhere in $\boldsymbol{R}$. A sufficient condition for the complete maximum principle is the existence of a so-called submarkovian resolvent $\left\{K_{p}\right\}(p>0)$, a family of positive kernels such that for each $p>0, K-K_{p}$ $=p K * K_{p}=p K_{p} * K$ and $p K_{p} * 1 \leqq 1$, and $K=K_{0}=\lim _{p \downarrow 0} K_{p}$. The outline of the proof: we have, for each $p>0$,

Received March 31, 1972. 


$$
K+\frac{1}{p} \varepsilon=\frac{1}{p}\left(\sum_{n=0}^{\infty}\left(p K_{p}\right)^{n}\right),
$$

where $\left(p K_{p}\right)^{0}=\varepsilon$, the Dirac measure on the origin, and $\left(p K_{p}\right)^{n}=$ $\left(p K_{p}\right)^{n-1} *\left(p K_{p}\right)$. This shows that $K+(1 / p) \varepsilon$ satisfies the complete maximum principle, so that $K$ itself satisfies the complete maximum principle.

\section{§2. Negative-definite functions.}

A complex-valued continuous function $\lambda(x)$ on $\boldsymbol{R}$ is said to be negativedefinite, when it holds that

i) $\lambda(0) \geqq 0, \lambda(-x)=\overline{\lambda(x)}$,

ii) for any $n$ points $x_{1}, x_{2}, \cdots, x_{n}$ on $\boldsymbol{R}$ and for any $n$ complex numbers $\xi_{1}, \xi_{2}, \cdots, \xi_{n}$ with $\sum_{j=1}^{n} \xi_{j}=0$,

$$
\sum_{j, k} \lambda\left(x_{j}-x_{k}\right) \xi_{j} \bar{\xi}_{k} \leqq 0
$$

As easily seen, $\lambda(x)=a x^{2}+i b x+c$ is negative-definite, if $a$ and $c$ are non-negative and $b$ is real. $\lambda(x)=c-(p-i x)^{-1}$ with $p>0$ and $c \geqq p^{-1}$ is also negative-definite, since

$$
-(p-i x)^{-1}=-\int_{0}^{\infty} e^{-p y} e^{i x y} d y .
$$

The sum of negative-definite functions $\lambda_{1}, \lambda_{2}, \cdots, \lambda_{n}$ is evidently negativedefinite. In this section we shall show that for specific $\lambda_{j}{ }^{\prime} \mathrm{s}, \lambda=\left(\sum_{j=1}^{n} \lambda_{j}{ }^{-1}\right)^{-1}$ is negative-definite.

LEMMA 1. Let $0<p_{1}<p_{2}<\cdots<p_{n}$ and $\mu_{1}, \mu_{2}, \cdots, \mu_{n}, \nu_{1}, \nu_{2}, \cdots, \nu_{n}$ be non-negative numbers, and let $f(z)$ be a complex-valued function $\left(\sum_{j=1}^{n}\left(\frac{\mu_{j}}{p_{j}+z}+\frac{\nu_{j}}{p_{j}-z}\right)\right)^{-1}$. If $\sum_{j=1}^{n} \mu_{j} \neq \sum_{j=1}^{n} \nu_{j}, f(z)$ is equal to

$$
a z+b+\sum_{k=1}^{2 n-1} \frac{c_{k}}{z-\alpha_{k}}
$$

with $b>0, \alpha_{k} c_{k}>0$. If $\sum_{j=1}^{n} \mu_{j}=\sum_{j=1}^{n} \nu_{j}$, it is equal to

$$
a z^{2}+b z+c+\sum_{k=1}^{2 n-2} \frac{d_{k}}{z-\alpha_{k}}
$$

with $a<0, c>0, \alpha_{k} d_{k}>0$. 
Proof. First we consider the case that $\sum_{j=1}^{n} \mu_{j} \neq \sum_{j=1}^{n} \nu_{j}$. In this case $f(z)$ is equal to $\prod_{j=1}^{n}\left(p_{j}^{2}-z^{2}\right) / \psi(z)$, where $\psi(z)=\sum_{k=1}^{n}\left(\left(\mu_{k}+\nu_{k}\right) p_{k}+\left(\nu_{k}-\mu_{k}\right) z\right)$ $\cdot \prod_{j \neq k}\left(p_{j}^{2}-z^{2}\right)$ is a polynomial of degree $2 n-1:$ the coefficient $c$ of $z^{2 n-1}$ is $(-1)^{n-1} \sum_{k=1}^{n}\left(\nu_{k}-\mu_{k}\right)$. Hence $f(z)$ is written as

$$
a z+b+\frac{\varphi(z)}{\psi(z)}
$$

with a polynomial $\varphi(z)$ of degree less than $2 n-1$ and $a=\left(\sum_{k=1}^{n}\left(\mu_{k}-\nu_{k}\right)\right)^{-1}$. Remark that $\psi(z)$ has $2 n-1$ simple zeros $\alpha_{k}$ 's and $\beta_{k}$ 's: in the case that $c$ is positive and $n$ is odd, or $c$ is negative and $n$ is even,

$$
\begin{aligned}
& \beta_{n}<-p_{n}<\beta_{n-1}<-p_{n-1}<\cdots<-p_{2}<\beta_{1}<-p_{1} \\
& p_{1}<\alpha_{1}<p_{2}<\cdots<p_{n-1}<\alpha_{n-1}<p_{n},
\end{aligned}
$$

and in the case that $c$ is negative and $n$ is odd, or $c$ is positive and $n$ is even

$$
\begin{aligned}
& -p_{n}<\beta_{n-1}<-p_{n-1}<\cdots<-p_{2}<\beta_{1}<-p_{1} \\
& p_{1}<\alpha_{1}<p_{2}<\cdots<p_{n-1}<\alpha_{n-1}<p_{n}<\alpha_{n} .
\end{aligned}
$$

In any case we have

$$
\begin{aligned}
& \frac{\varphi(z)}{\psi(z)}=\sum_{k} \frac{\varphi\left(\alpha_{k}\right)}{\psi^{\prime}\left(\alpha_{k}\right)} \frac{1}{z-\alpha_{k}}+\sum_{k} \frac{\varphi\left(\beta_{k}\right)}{\psi^{\prime}\left(\beta_{k}\right)} \frac{1}{z-\beta_{k}} \\
& \varphi(z)=\prod_{j=1}^{n}\left(p_{j}^{2}-z^{2}\right)-(a z+b) \psi(z) \\
& \frac{\varphi\left(\alpha_{k}\right)}{\psi^{\prime}\left(\alpha_{k}\right)}=\prod_{j=1}^{n} \frac{\left(p_{j}^{2}-\alpha_{k}^{2}\right)}{\psi^{\prime}\left(\alpha_{k}\right)}>0 \\
& \frac{\varphi\left(\beta_{k}\right)}{\psi^{\prime}\left(\beta_{k}\right)}=\prod_{j=1}^{n} \frac{\left(p_{j}^{2}-\beta_{k}^{2}\right)}{\psi^{\prime}\left(\beta_{k}\right)}<0 .
\end{aligned}
$$

Therefore $\alpha_{k} \varphi\left(\alpha_{k}\right) / \psi^{\prime}\left(\alpha_{k}\right)$ and $\beta_{k} \varphi\left(\beta_{k}\right) / \psi^{\prime}\left(\beta_{k}\right)$ are positive. We put $z=0$ in (1) and obtain

$$
b=\left(\sum_{j=1}^{n} \frac{\mu_{j}+\nu_{j}}{p_{j}}\right)^{-1}+\sum_{k=1}^{2 n-1} \frac{c_{k}}{\alpha_{k}}>0
$$

Now suppose that $\sum_{j=1}^{n} \mu_{j}=\sum_{j=1}^{n} \nu_{j}(\neq 0)$. Then 


$$
f(z)=a z^{2}+b z+c+\frac{\varphi(z)}{\psi(z)}
$$

with a polynomial $\varphi(z)$ of degree less than $2 n-1$ and $a=-\left(\sum_{k=1}^{n}\left(\mu_{k}+\nu_{k}\right) p_{k}\right)^{-1}$ $<0$. $\psi(z)$ has $2(n-1)$ simple zeros $\alpha_{k}$ 's and $\beta_{k}$ 's such that

$$
p_{k}<\alpha_{k}<p_{k+1}, \quad-p_{k+1}<\beta_{k}<-p_{k}(1 \leqq k \leqq n-1) .
$$

Similarly in the first case it holds that

$$
\begin{aligned}
& \frac{\varphi(z)}{\psi(z)}=\sum_{k=1}^{n-1} \frac{\varphi\left(\alpha_{k}\right)}{\psi^{\prime}\left(\alpha_{k}\right)} \frac{1}{z-\alpha_{k}}+\sum_{k=1}^{n-1} \frac{\varphi\left(\beta_{k}\right)}{\psi^{\prime}\left(\beta_{k}\right)} \frac{1}{z-\beta_{k}} \\
& \varphi(z)=\prod_{j=1}^{n}\left(p_{j}^{2}-z^{2}\right)-\left(a z^{2}+b z+c\right) \psi(z) \\
& \frac{\varphi\left(\alpha_{k}\right)}{\psi^{\prime}\left(\alpha_{k}\right)}=\prod_{j=1}^{n} \frac{\left(p_{j}^{2}-\alpha_{k}^{2}\right)}{\psi^{\prime}\left(\alpha_{k}\right)}>0 \\
& \frac{\varphi\left(\beta_{k}\right)}{\psi^{\prime}\left(\beta_{k}\right)}=\prod_{j=1}^{n} \frac{\left(p_{j}^{2}-\beta_{k}^{2}\right)}{\psi^{\prime}\left(\beta_{k}\right)}<0 .
\end{aligned}
$$

Hence the product of $\alpha_{k}$ and $d_{k}$ in (2) is positive for each $k$ and

$$
c=\left(\sum_{j=1}^{n} \frac{\mu_{j}+\nu_{j}}{p_{j}}\right)^{-1}+\sum_{k=1}^{2 n-2} \frac{d_{k}}{\alpha_{k}}>0 .
$$

By this lemma we have

Proposition 1. Let $p_{1}, p_{2}, \cdots, p_{n}, \mu_{1}, \mu_{2}, \cdots, \mu_{n}, \nu_{1}, \nu_{2}, \cdots, \nu_{n}$ be nonnegative numbers. Then

$$
\lambda(x)=\left(\sum_{j=1}^{n}\left(\frac{\mu_{j}}{p_{j}+i x}+\frac{\nu_{j}}{p_{j}-i x}\right)\right)^{-1}
$$

is negative-definite.

Proof. If $\sum_{j=1}^{n} \mu_{j} \neq \sum_{j=1}^{n} \nu_{j}$, we have by (1)

$$
\lambda(x)=i a x+b+\sum_{k=1}^{2 n-1} \frac{c_{k}}{i x-\alpha_{k}}
$$

with real $a \neq 0, b>0, \alpha_{k} c_{k}>0$. If $\sum_{j=1}^{n} \mu_{j}=\sum_{j=1}^{n} \nu_{j}(\neq 0)$,

$$
\lambda(x)=-a x^{2}+i b x+c+\sum_{k=1}^{2 n-2} \frac{d_{k}}{i x-\alpha_{k}}
$$


with real $b, a>0, c<0, \alpha_{k} d_{k}>0$. Therefore, in any case, $\lambda(x)$ is negative-definite.

\section{§3. Fractional powers of Heaviside kernel.}

Heaviside kernel $H$ is the convolution kernel on $\boldsymbol{R}$ induced by the function

$$
H(x)= \begin{cases}1, & x>0 \\ 0, & x \leqq 0\end{cases}
$$

Given a non-negative Lebesgue measurable function $f$, its Heaviside potential $H f(x)$ is

$$
H * f(x)=\int_{-\infty}^{x} f(y) d y .
$$

The kernel $H$ has a unique positive resolvent $\left\{H_{p}\right\}(p \geqq 0)$ such that

$$
H_{p}(x)=\left\{\begin{array}{ll}
e^{-p x}, & x>0 \\
0 & x \leqq 0
\end{array},\right.
$$

and $H$ satisfies the complete maximum principle. For a positive number $\alpha$ smaller than 1 , the fractional power $H^{\alpha}$ of $H$ is the convolution kernel defined by

$$
\frac{\sin \alpha \pi}{\pi} \int_{0}^{\infty} p^{-\alpha} H_{p} d p
$$

which is equal to the one induced by

$$
H^{\alpha}(x)=\left\{\begin{array}{ll}
\frac{x^{\alpha-1}}{\Gamma(\alpha)}, & x>0 \\
0 & x \leqq 0
\end{array},\right.
$$

In this section we shall show that for any $0<\alpha, \beta<1$, the sum of $H^{\alpha}$ and $\check{H}^{\beta}$ satisfies also the complete maximum principle, $\check{H}^{\beta}$ being the adjoint kernel of $H^{\beta}$, namely, $\check{H}^{\beta}(x)=H^{\beta}(-x)$. Notice that

$$
\check{H}^{\beta}(x)=\left\{\begin{array}{ll}
\frac{|x|^{\beta-1}}{\Gamma(\beta)}=\frac{\sin \beta \pi}{\pi} \int_{0}^{\infty} p^{-\beta} e^{p x} d p, & x<0 \\
0 & , \quad x \geqq 0
\end{array} .\right.
$$

Let $\mu$ and $\nu$ be positive Radon measures on $(0, \infty)$. We exclude the case $\mu=\nu=0$, and put 


$$
K(x)=\left\{\begin{array}{ll}
\int_{0}^{\infty} e^{-p x} d \mu(p), & x>0 \\
\int_{0}^{\infty} e^{p x} d \nu(p), & x<0
\end{array} .\right.
$$

We assume that $K(x)$ is locally Lebesgue integrable. Then it induces a positive convolution kernel $K$. Our aim is to prove the complete maximum principle for $K$. First we restrict ourselves to the case that $\mu$ and $\nu$ are measures on a bounded closed interval $[a, b](0<a<b<\infty)$. The Fourier transform of $K, \hat{K}(y)=\int_{-\infty}^{\infty} K(x) e^{-2 \pi i x y} d x$, is equal to

$$
\int_{a}^{b} \frac{d \mu(p)}{p+2 \pi i y}+\int_{a}^{b} \frac{d \nu(p)}{p-2 \pi i y}
$$

Hence $\lambda(y)=(K(y))^{-1}$ is continuous on $\boldsymbol{R}$, and

$$
\lambda(0)=\left(\int_{a}^{b} \frac{d \mu(p)}{p}+\int_{a}^{b} \frac{d \nu(p)}{p}\right)^{-1}>0, \quad \lambda(-y)=\overline{\lambda(y)} .
$$

LEMMA 2. $\lambda(y)$ is negative-definite.

Proof. We fix $n$ points $y_{1}, y_{2}, \cdots, y_{n}$ on the real line and $n$ complex numbers $\xi_{1}, \xi_{2}, \cdots, \xi_{n}$ such that $\sum_{j=1}^{n} \xi_{j}=0$. Given an arbitrary positive number $\varepsilon$, we can take a division of $[a, b], a=p_{0}<p_{1}<\cdots<p_{N}=b$, such that

$$
\left|\lambda\left(y_{j}-y_{k}\right)-\lambda_{\varepsilon}\left(y_{j}-y_{k}\right)\right|<\varepsilon(j, k=1,2, \cdots, n),
$$

where $\lambda_{\varepsilon}(y)=\left(\sum_{l=1}^{N}\left(\frac{\mu_{l}}{p_{l}+2 \pi i y}+\frac{\nu_{l}}{p_{l}-2 \pi i y}\right)\right)^{-1}$, and $\mu_{l}$ and $\nu_{l}$ denote respectively $\mu$-and $\nu$-masses of $l$-th subinterval of the division. Then it holds that

$$
\left|\sum_{j, k} \lambda\left(y_{j}-y_{k}\right) \xi_{j} \bar{\xi}_{k}-\sum_{j, k} \lambda_{s}\left(y_{j}-y_{k}\right) \xi_{j} \bar{\xi}_{k}\right| \leqq \varepsilon\left(\sum_{j}\left|\xi_{j}\right|\right)^{2},
$$

and that, $\lambda_{\varepsilon}(y)$ being negative-definite by Proposition $1, \sum_{j, k} \lambda\left(y_{j}-y_{k}\right) \xi_{j} \bar{\xi}_{k}$ $\leqq 0$.

This lemma leads us to the following

Proposition 2. If $\mu$ and $\nu$ are positive Radon measures on $[a, b]$ $(0<a<b<\infty)$, then the convolution kernel $K$ defined by 


$$
K(x)= \begin{cases}\int_{a}^{b} e^{-p x} d \mu(p), & x>0 \\ \int_{a}^{b} e^{p x} d \nu(p), & x<0\end{cases}
$$

satisfies the complete maximum principle.

Proof. It suffices to show the existence of a positive submarkovian resolvent $\left\{K_{q}\right\}(q \geqq 0)$ such that $K_{0}=K$. By the above proposition, $q+\lambda(y)$ is a negative-definite continuous function and $0<\lambda(0)=\left(\int_{a}^{b} \frac{d \mu(p)}{p}+\int_{a}^{b} \frac{d \nu(p)}{p}\right)^{-1}$ $\leqq \operatorname{Re} \lambda(y)$. Hence $(q+\lambda(y))^{-1}$ is a positive-definite continuous functions and by Bochner's theorem it is the Fourier transform of a positive measure $K_{q}$,

$$
(q+\lambda(y))^{-1}=\hat{K}_{q}(y)=\int_{-\infty}^{\infty} e^{-2 \pi i x y} d K_{q}(x)
$$

Notice that $|y K(y)|$ is bounded and hence every $K_{q}$ is a square integrable function. Therefore we have

$$
q \widehat{K}_{0} * K_{q}=q \hat{K}_{0} \hat{K}_{q}=\frac{q}{\lambda(y)(q+\lambda(y))}=\frac{1}{\lambda(y)}-\frac{1}{q+\lambda(y)}=\hat{K}_{0}-\hat{K}_{q},
$$

and hence $q K_{0} * K_{q}=K_{0}-K_{q}$. We have also $q \hat{K}_{q}(0)=\frac{q}{q+\lambda(0)}<1$.

By this proposition we have

THEOREM. Let $\mu$ and $\nu$ be positive Radon measures on $(0, \infty)$ such that the function $K$ defined by $(*)$ is locally Lebesgue integrable. Then $K$ satisfies the complete maximum principle.

Proof. $K$ is the limiting kernel of an increasing sequence of kernels discussed above. Hence our theorem follows immediately from the following lemma.

LEMMA 3. Let $\left\{K_{n}\right\}$ be an increasing sequence of positive kernels satisfying the complete maximum principle. If $K=\lim K_{n}$ defines a positive kernel, it satisfies the complete maximum principle.

Poof. Let $f$ and $g$ be essentially bounded positive Lebesgue measurable functions vanishing outside a compact set such that $K f \leqq K g+1$ on $S f=\{x \in \boldsymbol{R} ; f(x)>0\}$. We shall prove the inequality $K f \leqq K g+1$ 
almost everywhere in $\boldsymbol{R}$. Without loss of generality, we may suppose that $K f<K g+1$ on $S f$. Let $f_{n}$ be the restriction of $f$ to the set $\left\{x \in S f ; K f \leqq K_{n} g+1\right\}$. Then $\left\{f_{n}\right\}$ increases to $f$. Since $K_{n}$ satisfies the complete maximum principle, we have $K_{n} f_{n} \leqq K_{n} g+1 \leqq K g+1$ almost everywhere. Noticing that $\left\{K_{n} f\right\}$ increases to $K f$, we conclude that $K f=\lim K_{n} f_{n}$, and $K f \leqq K g+1$.

Setting $\mu=\frac{\sin \alpha \pi}{\pi} p^{-\alpha}$ and $\nu=\frac{\sin \beta \pi}{\pi} p^{-\beta}$, we have

COROLLARY. $H^{\alpha}+\check{H}^{\beta}$ satisfies the complete maximum principle, for any $0<\alpha, \beta<1$.

Nagoya University 